\title{
Localization of Mobile Robot Based on Least Square Method
}

\author{
Li Jialu \\ Tianjin Key Laboratory for Control Theory \& \\ Applications in Complicated Systems \\ Tianjin, China \\ lijialu_1989@163.com
}

\author{
Long Linbing, Zhang Baofeng \\ Tianjin Key Laboratory for Control Theory \& \\ Applications in Complicated Systems \\ Tianjin, China \\ zhangbaofeng@263.com
}

\begin{abstract}
Localization is the basis for navigation of mobile robots. This paper focuses on key techniques of localization for mobile robots based on vision. Firstly, the specific measures and steps of the algorithm are analyzed and researched in depth. In the study, SIFT algorithm combined with epipolar geometry constraint is used on the environment feature point detection, matching and tracking. And the method of RANSAC combined with the least squares is used to obtain accurate results of the motion estimation. Then the necessary experiments are carried out to verify the correctness and effectiveness of algorithms. The experimental results verified the accuracy of the improved algorithm.
\end{abstract}

Keywords-SIFT; RANSAC; motion estimation; least squares

\section{INTRODUCTION}

In recent years, with the continuous development of science and technology, the mobile robot is more widely used in various fields of the national economy, such as industrial, military, defense, rescue, planetary exploration. Growing number of researchers have begun to carry out the research work of the robot, which the mobile robot technology is increasingly becoming a hot spot.

Autonomous navigation technology let the mobile robot has broad application prospects more than the average robot, which has a very important scientific significance. To achieve the robot autonomous navigation is necessary to solve the three problems [1]: (1) robot self-localization problem, (2) robot target detection problem, (3) robot target detection problem. Among them, the self-positioning of the robot is the basis of the latter two problems, only to get the accurate positioning results in order to guarantee the accuracy of autonomous navigation. Commonly used positioning method is based on the visual, which is not only able to make up for the deficiencies of the traditional positioning methods, but also has the advantages of low power consumption, broad range of applications, small devices [2].

This paper studies the positioning of mobile robots based on stereo vision. In this article, SIFT algorithm is used on the environment feature point detection, matching and tracking, which can achieve the accurate identification of the target in many circumstances. The search range can be reduced to the epipolar line by epipolar geometry constraint is combined with the SIFT algorithm, which can increase the accuracy of matching and reduce the error. In the motion estimation, the method of RANSAC combined with the least squares is used to reduce the error of the image processing and easier to implement fast, accurate, and robust motion estimation.

\section{ALGORITHM}

Vision-based mobile robot localization method is as follows: first to detection, matching and tracking feature point, and reconstruction of three-dimensional coordinates. Then combined with motion estimation algorithm, to arrive at more accurate position of the robot. The most critical steps in the entire process are feature extraction, feature matching and tracking, and the motion estimation.

\section{A. Feature Extraction}

Common feature point extraction algorithm mainly includes Harris corner detection [3], SIFT detection [4], and fast algorithms SURF [5] and CenSurE [6]. These types of feature point extraction algorithms were respectively achieved and compared during the experiment. Wherein, SIFT is better than the other algorithms on the number and accuracy of the extracted feature points. Therefore, in subsequent experiments, using the SIFT feature extraction results.

\section{B. Feature Matching and Tracking}

The feature points, which were extracted in the previous step, are matched by Euclidean distance of SIFT feature vectors. SIFT operator is stable to noise and invariant to scaling, rotation, translation and illumination in matching images [7]. The above characteristics are suitable for the study of mobile robot localization. A 128-dimensional SIFT feature vector can be formed after feature extraction [8]. The Euclidean distance between the left and right image feature vector as a similarity measure. Calculating the proportion of the nearest Euclidean distance and the next closest, if the ratio was smaller than a certain threshold ratio, then the match is successful. in the matching process, matching rule constraints and the appropriate ratio threshold can be used to determine the matching points.

Feature tracking algorithm is similar to feature matching algorithm, except that the search range is more than confined in the same horizontal line, but in a window area. This paper selects epipolar geometric constraints to narrow the search to epipolar line, which can increase the accuracy of matching.

\section{Motion Estimation}

Motion estimation can obtain the robot motion parameters by processing the fore and after image pairs, and its robustness is considerable as well when the robot runs a 
long distance. The motion parameters of the robot is given by (1):

$$
P_{c j}=R P_{p j}+T
$$

where $P_{c j}$ and $P_{p j}$ are the 3-D coordinates of the $j$ feature points of the fore and after frame respectively, matrix $R$ and vector $T$ stands for the rotatable parameters and translation parameters of the robot [9].

In order to give attention to both real-time and accuracy of the algorithm, using the following methods: The RANSAC combined with linear least square method to obtained estimates of the robot motion parameters, then the estimated values are substituted into the nonlinear least squares method to find the precise estimate of the robot motion parameters.

First, the rotation matrix $R$ and translation vector $T$ are 3 $\times 3$ and $3 \times 1$ respectively, a total of 12 unknowns, so theoretically requires at least four non-collinear matching points substituting into (1) to be calculated. Solving the least squares solution makes $\left\|P_{c j}-\left(R P_{p j}+T\right)\right\|^{2}$ minimum. The number of the fore and after image pairs which were matched is $n$. Using RANSAC algorithm to calculate for several times can effectively except exterior points, and get a collection of $n^{\prime}$ interior points. Meanwhile, a more accurate estimate is obtained, we put this estimate as a iterative initial value of the nonlinear least squares algorithm. When solving linear least squares solution, using the method of singular value decomposition to avoid obtaining reflected rotation matrix [4] and enhance the anti-noise capability. Specific methods are as follows:

$$
\begin{gathered}
\mu_{p}=\frac{1}{n} \sum_{i=1}^{n} P_{p i}, \\
\mu_{c}=\frac{1}{n} \sum_{i=1}^{n} P_{c i}, \\
\sum_{c p}=\frac{1}{n} \sum_{i=1}^{n}\left(P_{c i}-\mu_{c}\right)\left(P_{p i}-\mu_{p}\right) .
\end{gathered}
$$

Using singular value decomposition for the covariance matrix $\sum_{c p}, \sum_{c p}=U D V$, and because

$$
S=\left\{\begin{array}{cc}
D=\operatorname{diag}\left(d_{i}\right), & d_{1} \geq d_{2} \ldots \geq d_{m} \geq 0 \\
I, & \operatorname{det}\left[\sum_{c p}\right] \geq 0 \\
\operatorname{diag}(1,1,1, \ldots,-1), & \operatorname{det}\left[\sum_{c p}\right]<0
\end{array},\right.
$$

the rotation matrix $R$ can be expressed as follow:

$$
R=U S V,
$$

which combines (1) can be drawn the vector $T$.

Next, using the Levenberg-Maquardt algorithm for nonlinear least squares estimation to get an accurate estimate result. The first step, put the rotation matrix $R$ rewritten into quaternion form. Let $q=\left[q_{0}, q_{1}, q_{2}, q_{3}\right]^{T}, q_{0}{ }^{2}+q_{1}{ }^{2}+q_{2}{ }^{2}+q_{3}{ }^{2}=1$, then

$$
\begin{gathered}
R= \\
{\left[\begin{array}{ccc}
q_{0}^{2}+q_{1}^{2}-q_{2}^{2}-q_{3}^{2} & 2\left(q_{1} q_{2}-q_{0} q_{3}\right) & 2\left(q_{1} q_{3}+q_{0} q_{2}\right) \\
2\left(q_{1} q_{2}+q_{0} q_{3}\right) & q_{0}^{2}-q_{1}^{2}+q_{2}^{2}-q_{3}^{2} & 2\left(q_{2} q_{3}-q_{0} q_{1}\right) \\
2\left(q_{1} q_{3}-q_{0} q_{2}\right) & 2\left(q_{2} q_{3}+q_{0} q_{1}\right) & q_{0}^{2}-q_{1}^{2}-q_{2}^{2}+q_{3}^{2}
\end{array}\right]}
\end{gathered}
$$

The minimum of $x$ in $F(x)=\sum_{j=1}^{n^{\prime}}\left\|P_{c j}-f_{j}(x)\right\|^{2}$ will be obtained by the iterative method of Levenberg-Maquardt algorithm, and then the value of the rotational and translational will be drawn. Where $f_{j}(x)=R(x) P_{p j}+T$, the initial value of $x$ is derived by the linear least squares method in the previous step.

The iterative process is as follows:

a) Define the following variable:

$$
\begin{gathered}
A_{k}=\sum_{j=1}^{n^{\prime}}\left(\frac{\partial f_{j}\left(x_{k}\right)}{\partial x_{k}}\right)^{T}\left(\frac{\partial f_{j}\left(x_{k}\right)}{\partial x_{k}}\right), \\
b_{k}=-\sum_{j=1}^{n^{\prime}}\left(\frac{\partial f_{j}\left(x_{k}\right)}{\partial x_{k}}\right)^{T}\left(P_{c j}-f_{j}\left(x_{k}\right)\right) ;
\end{gathered}
$$

b) Seek the solution of the following equation:

$$
\left(A_{k}+\alpha_{k} I\right) d_{k}=b_{k}
$$

c) Obtain the search direction $d_{k}$, and where

$$
x_{k+1}=x_{k}+d_{k}
$$

d) If $F\left(x_{k+1}\right)<F\left(x_{k}\right)$, go to step $f$ ), otherwise go to step e);

e) If $\left|\frac{F\left(x_{k+1}\right)-F\left(x_{k}\right)}{F\left(x_{k}\right)}\right|<\varepsilon$, output $x=x_{k}$, else set $\alpha=\beta \alpha$ and go to step $a$ );

f) If $\left|\frac{F\left(x_{k+1}\right)-F\left(x_{k}\right)}{F\left(x_{k+1}\right)}\right|<\varepsilon$, output $x=x_{k+1}$, else set $\alpha=\frac{\alpha}{\beta}$ and go to step $a$ ).

$f(x)$ is a matrix-valued function of $x$ in $d$ ) to $f$ ), so 


$$
\frac{\partial f(x)}{\partial x}=\left[\begin{array}{ccc}
\frac{\partial f_{1}(x)}{\partial x_{1}} & \cdots & \frac{\partial f_{1}(x)}{\partial x_{7}} \\
\vdots & \ddots & \vdots \\
\frac{\partial f_{3}(x)}{\partial x_{1}} & \cdots & \frac{\partial f_{3}(x)}{\partial x_{7}}
\end{array}\right] .
$$

In the step $c$ ), the previous four variables of $x$ should be subjected to the normalization processing. The value of $\alpha, \beta$ and $\varepsilon$ are empirical values, which can be adjusted depending on the application environment and precision.

\section{EXPERIMENTS AND RESULTS}

In order to test the accuracy and robustness of this method when the robot runs a long distance, we carried out experiments in outdoor environment. First, extract the feature points of the captured images by SIFT algorithm. Then, match and track these feature points through the method of SIFT algorithm combined with epipolar geometry constraint. Last, calculate the motion parameters of the robot through the method of RANSAC combined with the least squares. The matching result of feature tracking is shown in Fig. 1, the comparison between the calculation results based on RANSAC algorithm and the true path is represented in Fig. 2, and Fig. 3 demonstrates the error.

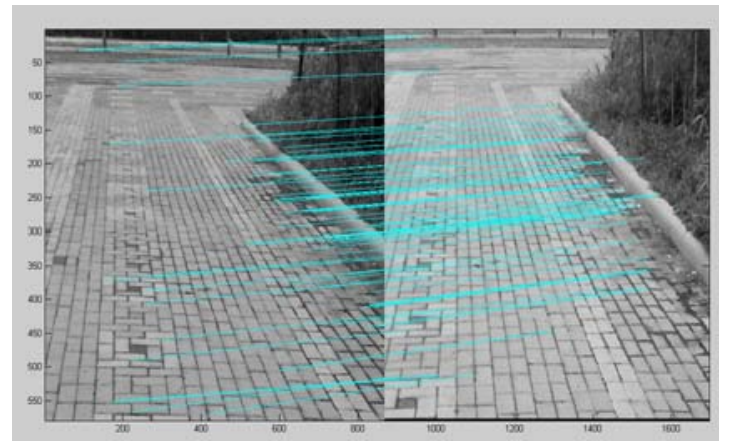

Figure 1. Results of feature extracting and matching

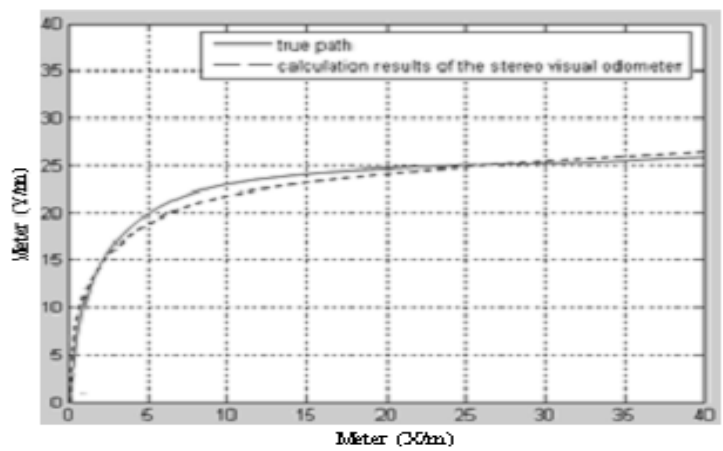

Figure 2. Contrast between the calculation results of the stereo visual odometer and the true path

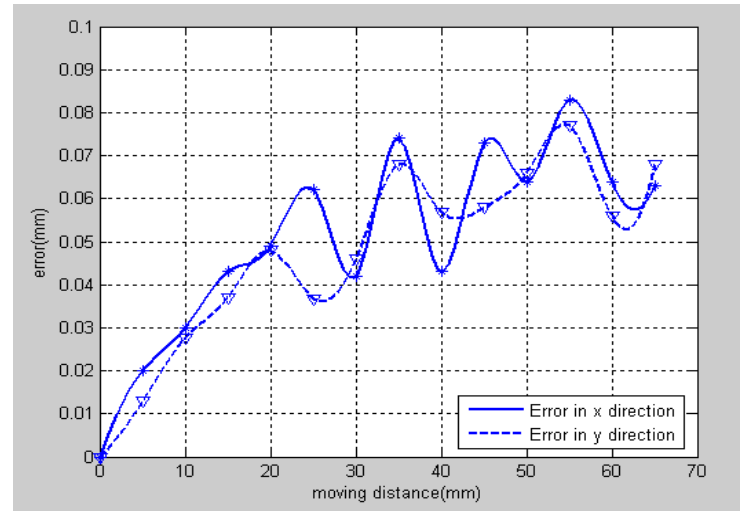

Figure 3. Error of the stereo visual odometer

In Fig. 2, solid line stands for the true path and solid point is the robot motion estimate. From the result and error shown in the figures above, we can conclude that the calculation results during the long distance moving is quite accurate, and the relative error is no more than $8 \%$, at the same time the error does not take on a nonlinear growth trend with the movement distance increasing when the robot takes long roaming.

\section{CONCLUSIONS}

The article describes the algorithm of the mobile robot localization, mainly include feature extraction, feature matching and tracking, and motion estimation. And on this basis, the method of RANSAC combined with the least squares is proposed to optimize the results of the motion estimation. Multiple outdoor experiments were done. Experimental results show that the method of mobile robot localization based on RANSAC algorithm can get more accurate motion parameters, and the measurement error dose not increase with the movement distance increasing. Gradual improvement for this method should be done in future research to better used in the Autonomous navigation technology.

\section{ACKNOWLEDGMENT}

This work was financially supported by the National Natural Science Foundation (grant: F010404 (61172185)) and National Undergraduate Scientific and Technological Innovation Project.

\section{REFERENCES}

[1] P. Goel, S. L. Roumeliotis, and G. S. Sukhatme, "Robust localization using relative and absolute position estimates," Proc. IEEE Symp. Intelligent Robots and Systems (IROS 99), IEEE Press, Nov. 1999, pp. 1134-1140.

[2] Li Yongjia, "Research on Robot Localization Based on Vision," unpublished.

[3] C. Harris, M. Stephens, "A combined corner and edge detector," Fourth Alvey Vision Conference, 1988, pp. 147-151.

[4] D. G. Lowe, "Distinctive image features from scale-invariant keypoints,” Computer Vision, vol. 60, Dec. 2004, pp. 91-110. 
[5] H. Bay, A. Ess, T. Tuytelaars, and L. V. Gool, "Speeded up robust features," Computer Vision and Image Understanding, vol. 110, Aug. 2008, pp. 346-359.

[6] M. Agrawal, K. Konolige, and M. R. Blas, "Center surround extremas for realtime feature detection and matching," Proc. IEEE Symp. Computer Vision (ECCV 08), IEEE Press, Oct. 2008, pp. 189-224.

[7] J. Sun, H. Y. Shum, and N. N. Zheng, "Stereo matching using belief propagation,” Proc. IEEE Symp. Pattern Analysis and Machine Intelligence (TPAMI 03), IEEE Press, Jul. 2003, pp. 787-800.
[8] T. Lindeberg, "Scale-space theory:A basic tool for analyzing structure at different scales,” Applied Statistics, vol. 21, Nov. 1994, pp. 224270 .

[9] B. Kitt, A. Geiger, and H. Lategahn, "Visual odometry based on stereo image sequences with RANSAC-based outlier rejection scheme,” Proc. IEEE Symp. Intelligent Vehicles Symposium (ITSS 10), IEEE Press, Dec. 2010, pp. 256-270. 